\title{
ADEQUACY OF HOSPITAL DISCHARGE LETTERS FROM OLDER ADULT MENTAL HEALTH (OAMH) WARDS IN A SCOTTISH HEALTH REGION
}

\author{
Gary Stevenson, Charlie Guy, Shepherd Chigwada \& Maria Tywoniuk \\ Department of Psychiatry, NHS Fife, Fife, Scotland KY15 5RR (gstevenson@nhs.net)
}

\section{Background \& Objectives}

- The safe clinical management of patients can depend on timely and accurate transfer of information between treating clinicians.

- $\quad$ Failure to observe such clinical standards may disadvantage patients and be a focus of medico-legal enquiry.

- This study examined the adequacy of clinical discharge letters from the OAMH inpatient wards in one Scottish Health region (Fife, population 365,000), with focus on medication advice, follow-up arrangements and information transfer interval

- $\quad$ To recommend any improvements to the information transfer on hospital discharge letters.

\section{Methods}

All discharge notifications from the five OAMH wards were examined retrospectively for the 18-month period ending August 2018, and examined against the electronic records and case-files.

\section{Results}

- 518 discharge notifications were reduced to 298 after removing duplicates, inter-ward transfers and deaths.

- Female:male ratio of 1.17:1; average age 76 (range 52-100) years old

- Average inpatient duration 122 days, range 1-1858 days

- No identified discharge letter in $17.1 \%$.

- $42 \%$ patients were discharged to ( $12 \%$ admitted from) residential care.

- $35 \%$ patients had dementia with $43 \%$ of them being discharged on antipsychotics without mention of prescribing restrictions or risks.

- $35 \%$ of all patients were discharged on antipsychotics with no advice on monitoring in $93 \%$.

- Guardians/Powers of attorney were rarely mentioned.

- The 247 discharge letters took 43 (range 1-345) days to verify, $52 \%$ only verified after one month.

- For the 19 inpatients with admissions greater than one year, only $11(58 \%)$ had verified discharge letters, and then on average 64 days post discharge

\section{Conclusions}

- There appear significant failings in the timely transfer of clinical details between OAMH inpatient services and primary care services in this region that requires urgent intervention to minimise clinical risk and maximise patient safety.

- An updated discharge letter format and monitoring processes have been proposed and will be subject to review. 